\title{
The Survey of Malnutrition Prevalence in Patients With Parkinson Disease In Tehran, Iran
}

\author{
Koorosh Etemad ${ }^{1}$, Farzad Ashrafi' ${ }^{2}$, Amir Shahbazzadeh ${ }^{3}$, Tara Moghaddasfar ${ }^{3}$, Mehran Arab Ahmadi $^{1,2^{*}}$ \\ ${ }^{1}$ School of Public Health, Shahid Beheshti University of Medical Sciences, Tehran, Iran \\ ${ }^{2}$ Functional Neurosurgery Research Center, Shahid Beheshti University of Medical Sciences, Tehran, Iran \\ ${ }^{3}$ School of Medicine, Shahid Beheshti University of Medical Sciences, Tehran, Iran
}

\begin{abstract}
Background: Parkinson disease (PD) introduced as the second most prevalent neurodegenerative disease following Alzheimer disease. A patient's nutritional status may be compromising in PD. This study aimed to describe the outpatient nutritional status in patients with PD at a third-level hospital in Iran.

Methods: The validated Persian version of the Mini-Nutritional Assessment (MNA) questionnaire was used to evaluate the nutritional status of 158 Iranian PD patients (age, 35-85 years). The exclusion criteria were as follows: chronic comorbidities affecting the nutritional status (e.g., diabetes and hypertension); adherence to special diets; and cognitive disorders. Based on the MNA questionnaire, a total score below 17 represented malnutrition, while scores $17-24$ indicated a risk of malnutrition. Results: Based on the findings, $26 \%$ of the participants had low weight, $67 \%$ of whom were over 65 years. According to the MNA questionnaire, $14.3 \%$ of the participants showed malnutrition and $26.2 \%$ were at risk of malnutrition.

Conclusion: Based on our findings, PD can contribute to reduced nutritional status. More than onefourth of the PD population was at risk of malnutrition, which highlights the need for more attention towards nutritional assessment. Nutritional assessment is essential for the development of nutritional interventions and early detection of PD patients who are at risk of malnutrition.

Keywords: Parkinson's disease; Prevalence; Nutritional status.
\end{abstract}

*Correspondence to Mehran Arab Ahmadi, MD; Functional Neurosurgery Research Center, Shahid Beheshti University of Medical Sciences, Tehran, Iran Tel: +98 9128347682, Email:

mehran_arabahmadi@yahoo. com

Published online 30 September 2018

Citation: Etemad K, Ashrafi F, Shahbazzadeh A, Moghaddasfar T, Arab Ahmadi M. The survey of malnutrition prevalence in patients with Parkinson disease in Tehran, Iran. Int Clin Neurosci J. 2018;5(3):103-105. doi:10.15171/icnj.2018.20

\section{Introduction}

Parkinson disease (PD) is a result of the progressive degeneration of dopaminergic neurons in the brain and a group of monoaminergic cells in brain tissues. This disease is recognized as the second most common neurodegenerative disorder following Alzheimer's disease, affecting $1 \%$ of the population over 65 years worldwide, with an incidence of 14-100 cases per 100000 each year. ${ }^{1}$

PD patients face difficulties, such as motor dysfunctions, including resting tremors and postural instability, as well as non-motor symptoms, such as depression, changes in taste, gastrointestinal dysfunction, and various eating problems (e.g., swallowing dysfunction and chewing). ${ }^{2}$ The objective of PD treatment is to increase dopamine levels, in addition to alleviating the symptoms, for which medications such as levodopa utilized. ${ }^{3}$

Nutritional status majorly influences an individual's quality of life and affects everyday activities in the elderly.
Nutritional status may become compromised in patients with PD, usually by the loss of weight. The possible weight loss factors in these patients are low dietary intake due to dysphagia or anorexia, a lowering of absorption caused by slow gastric emptying, and an increase in energy consumption due to high muscular activity. ${ }^{5}$ Moreover, the disease itself and the side effects of medications may be involved, as well. ${ }^{6}$

Malnutrition associated with the inadequate intake of proteins, calories, and other nutrients, which are necessary for the repair or maintenance of body tissues. Accurate diagnosis of malnutrition and proper interventions should be prioritized to delay or prevent poor outcomes, including more prolonged recovery, reduced quality of life, and increased risk of falls, osteoporosis, and hospitalization..$^{7-10}$ Therefore, correct nutrition plays a significant role in the prevention of malnutrition and its associated complications in $\mathrm{PD}$ patients.

The objective of this study was to describe the 
nutritional status of ambulatory patients with PD in a third-level hospital.

\section{Methods}

This descriptive study was conducted among adults (age, 35-85 years) with idiopathic PD from the Outpatient Movement Disorders Clinic of Shohada hospital in Tehran during 2017. In the primary evaluation of patients, after demographic data, including clinical history, gender, and age, were collected, initial symptoms, disease duration, dietary habits (using a 24-hour reminder), and anthropometric indices (weight, size, body mass index [BMI], mid-upper arm muscle area [(M) UAMA], and mid-upper arm circumference [(M) UAC]) were determined.

Nutritional status assessed with the Mini-Nutritional Assessment (MNA) test, which includes simple measurements and brief questions; it took nearly 10-15 minutes to complete the test. The complete version of the MNA questionnaire consists of two sections and 18 items, i.e., six screening and 12 assessment questions in section I and section II, respectively. The maximum score is 30 in the questionnaire (16 for screening questions and 14 for assessment questions). A total score of $<17$ was indicative of malnutrition, scores 17-23.5 signified the risk of malnutrition and scored $\geq 24$ represented a normal nutritional status15. In this study, the full translated form of MNA questionnaire, which was provided by Nestlé Nutrition Institute, was applied 11-12. Subsequently, data assessed in SPSS 20 (Chicago, IL, USA).

\section{Results}

A total of 158 patients ( $44 \%$ male) were studied, and an average disease duration of 6.44 years identified. Overall, $72 \%$ of the patients reported tremor as an initial symptom. The subjects had a mean weight of $72.45 \pm 12.5 \mathrm{~kg}$, mean age of $67.42 \pm 7.5$ years, mean height of $164 \pm 8 \mathrm{~cm}$, and mean BMI of $26.2 \pm 4.46 \mathrm{~kg} / \mathrm{m}^{2}$. Twenty-six percent of the population identified as having low weight, and $32 \%$ identified as overweight or obese.

Considering the MUAC, which was $13.47 \pm 7.41 \mathrm{~mm}$, $26 \%$ of the studied subjects had a reduced fat mass. In addition to $\mathrm{PD}, 46 \%$ and $37.4 \%$ of the population presented hypertension and diabetes, respectively. Table 1 shows the clinical and anthropometric characteristics of the subjects classified by age (under 50 years and over 50 years). Table 2 demonstrates the data from MNA test of PD patients; overall, $14.3 \%$ had malnutrition, and $26.2 \%$ were at risk of malnutrition.

\section{Discussion}

Nutritional status defined as the balance between food intake and its use by the body. ${ }^{10}$ In this study, it observed that $26 \%$ of the population was underweight, of whom

Table 1. Demographic and Anthropometric Characteristics of Patients With Parkinson Disease

\begin{tabular}{|c|c|c|c|}
\hline Characteristics & Parkinson Disease Patients, Age $<65(n=52)$ & Parkinson Disease Patients, Age $>65(n=106)$ & $P$ Value \\
\hline Gender, No. (\%) & & & 0.745 \\
\hline Female & $26(50)$ & $58(55)$ & \\
\hline Male & $26(50)$ & $47(45)$ & \\
\hline Level of education, No. (\%) & & & 0.421 \\
\hline Illiterate & $6(11.5)$ & $24(23.2)$ & \\
\hline Primary & $10(19.2)$ & $21(19.2)$ & \\
\hline High school & $23(12)$ & $38(36.5)$ & \\
\hline College & $24(46.1)$ & $23(21.1)$ & \\
\hline Weight (kg), mean (SD) & $71.2(14.3)$ & $68.2(12.6)$ & 0.227 \\
\hline $\mathrm{BMI}\left(\mathrm{kg} / \mathrm{m}^{2}\right)$, mean $(\mathrm{SD})$ & $24.8(2.37)$ & $23.9(2.46)$ & 0.902 \\
\hline Low weight & 13 & 32 & \\
\hline Normal & 32 & 64 & \\
\hline Overweight & 5 & 7 & \\
\hline Obese & 2 & 3 & \\
\hline $\mathrm{MAC}(\mathrm{cm})$, mean $(\mathrm{SD})$ & $28.8(5.1)$ & $27.2(3.9)$ & 0.033 \\
\hline $\mathrm{CC}(\mathrm{cm})$, mean $(\mathrm{SD})$ & $33.6(2.8)$ & $31.4(2.1)$ & 0.605 \\
\hline
\end{tabular}

Abbreviations: BMI, body mass index; MAC, mid-arm circumference; CC, calf circumference.

Table 2. Mini-Nutritional Assessment Findings and Nutritional Status

\begin{tabular}{|c|c|c|c|}
\hline Characteristics & Parkinson Disease Patients, Age $<65(n=52)$ & Parkinson Disease Patients, Age $>65(n=106)$ & $P$ Value \\
\hline Score, mean (SD) & $15.1(2.25)$ & $13.2(3.15)$ & 0.006 \\
\hline Malnourished (\%) & 4.3 & 12 & 0.037 \\
\hline At risk of malnutrition (\%) & 13.2 & 2.4 & $0.004^{*}$ \\
\hline Normal nutritional status. $(\%)$ & 82.5 & 63.6 & 0.822 \\
\hline
\end{tabular}

Abbreviations: BMI, body mass index; MAC, mid-arm circumference; CC, calf circumference; 
$66 \%$ were older than 50 years. Different studies have reported that the prevalence of malnutrition due to the deficiency in patients with PD ranges from 3\% to $60 \%$. 14-15 In our study, according to the MNA questionnaire, patients were considered at risk of malnutrition (13.3\%) or had malnutrition (26.7\%).

In most cases, the progression of PD accompanied by a consequent loss of weight.14-16 In this study, 69.4\% of the patients reported having lost weight in the last three months, and most subjects older than 50 years (65\%) reported a decrease in food intake in the last week. All subjects have treated with levodopa, which associated with an increased risk of malnutrition risk. ${ }^{6-15}$

In comparison with men, women usually are exposed to a higher risk of malnutrition and unintentional weight $\operatorname{loss}^{17}$; this finding has confirmed in previous studies. ${ }^{1,2}$ This study also aimed to categorize and compare malnutrition in younger and older subjects. However, further studies are necessary to determine the critical risk factors for each age group. Another strength of this study is the use of a nutritional assessment tool (Persian version of the MNA questionnaire), which applies to any age and includes recent weight changes and physical examination. This study also revealed that in this population, anthropometric indices alone could not detect malnourishment.

In conclusion, this study showed that $26.7 \%$ of patients had malnutrition. Together with the findings of other studies, this indicates that PD can contribute to a decrease in the nutritional status. Therefore, nutritional assessment is essential for early detection of patients with PD at risk of malnutrition. Also, implementation of individualized nutritional interventions and the development of strategies, which favor food intake to improve the nutritional status and general condition of patients with idiopathic PD, should be highlighted.

Nutritional evaluation, education, and even interventions should be considered in the multidisciplinary work-up of PD patients to improve their nutrition and to prevent weight loss or to worsening of nutritional status. Moreover, a follow-up examination of PD patients to track longitudinal changes in the nutritional status is of high demand to better understand the factors influencing nutrition; this could investigate in future studies.

\section{Conflict of Interest Disclosures}

The authors declare that they have no conflict of interests.

\section{Ethical Statement}

The Ethics Committee of Shahid Beheshti University of Medical Sciences approved the study protocol. Also, written informed consents obtained after the participants were informed about the aims and objectives of the study.

\section{Acknowledgments}

The authors would like to thank all the participants and their families who participated in the current study. This article extracted from the thesis written by Mehran Arab Ahmadi for the degree of Master of public health.

\section{References}

1. Ulamek-Koziol M, Bogucka-Kocka A, Kocki J, Pluta R. Good and bad sides of diet in Parkinson's disease. Nutrition. 2013;29(2):474-5. doi: 10.1016/j.nut.2012.09.013.

2. Gaba A. Recent studies on nutrition and Parkinson's disease prevention: a systematic review. Open J Prev Med. 2015;5(5):197-205. doi: 10.4236/ojpm.2015.55023.

3. Uc EY, Struck LK, Rodnitzky RL, Zimmerman B, Dobson J, Evans WJ. Predictors of weight loss in Parkinson's disease. Mov Disord. 2006;21(7):930-6. doi: 10.1002/mds.20837.

4. Neumann SA, Miller MD, Daniels L, Crotty M. Nutritional status and clinical outcomes of older patients in rehabilitation. J Hum Nutr Diet. 2005;18(2):129-36. doi: 10.1111/j.1365277X.2005.00596.x.

5. Wang G, Wan Y, Cheng Q, Xiao Q, Wang Y, Zhang J, et al. Malnutrition and associated factors in Chinese patients with Parkinson's disease: Results from a pilot investigation. Parkinsonism Relat Disord. 2010;16(2):119-23. doi: 10.1016/j.parkreldis.2009.08.009.

6. Laudisio A, Vetrano DL, Meloni E, Ricciardi D, Franceschi F, Bentivoglio AR, et al. Dopaminergic agents and nutritional status in Parkinson's disease. Mov Disord. 2014;29(12):15437. doi: 10.1002/mds.25991.

7. Sheard JM, Ash S, Silburn PA, Kerr GK. Prevalence of malnutrition in Parkinson's disease: a systematic review. Nutr Rev. 2011;69(9):520-32. doi: 10.1111/j.17534887.2011.00413.x.

8. Correia MI, Waitzberg DL. The impact of malnutrition on morbidity, mortality, length of hospital stay and costs evaluated through a multivariate model analysis. Clin Nutr. 2003;22(3):235-9. doi: 10.1016/S0261-5614(02)00215-7.

9. Crogan NL, Pasvogel A. The influence of protein-calorie malnutrition on quality of life in nursing homes. J Gerontol A Biol Sci Med Sci. 2003;58(2):159-64.

10. Banks M, Bauer J, Graves N, Ash S. Malnutrition and pressure ulcer risk in adults in Australian health care facilities. Nutrition. 2010;26(9):896-901. doi: 10.1016/j.nut.2009.09.024.

11. Sadrzadeh-Yeganeh $H$, Angoorany P, Keshavarz SA, Rahimi A, Ahmady B. Comparison of two nutrition education techniquies on breakfast-eating practice in primary school girls, Tehran. Journal of School of Public Health and Institute of Public Health Research. 2006;4(1):65-72.

12. Jamaati HR, Pajouh P, Nayebi M, Baghaie N, Baghaie N, Khosravani H. Ascorbic acid concentration in plasma and white blood cells of patients with bronchial asthma. Tanaffos. 2006;5(4):29-35. [Persian]

13. Amirkalali B, Sharifi F, Fakhrzadeh $H$, Mirarefin $M$, Ghaderpanahi M, Larijani B. Evaluation of the Mini Nutritional Assessment in the elderly, Tehran, Iran. Public Health Nutr. 2010;13(9):1373-9. doi: 10.1017/s1368980010000303.

14. Barichella M, Cereda E, Pezzoli G. Major nutritional issues in the management of Parkinson's disease. Mov Disord. 2009;24(13):1881-92. doi: 10.1002/mds.22705.

15. Lorefalt B, Granerus AK, Unosson M. Avoidance of solid food in weight losing older patients with Parkinson's disease. J Clin Nurs. 2006;15(11):1404-12. doi: 10.1111/j.13652702.2005.01454.x.

16. Barichella M, Marczewska A, Vairo A, Canesi M, Pezzoli G. Is underweightness still a major problem in Parkinson's disease patients? Eur J Clin Nutr. 2003;57(4):543-7. doi: 10.1038/ sj.ejcn. 1601581.

17. Sheard JM, Ash S, Mellick GD, Silburn PA, Kerr GK. Malnutrition in a sample of community-dwelling people with Parkinson's disease. PLoS One. 2013;8(1):e53290. doi: 10.1371/journal.pone.0053290. 